International Journal of Engineering \& Technology, $7(4.5)(2018) 428-430$
International Journal of Engineering \& Technology
SPC
Website: www.sciencepubco.com/index.php/IJET
Research paper

\title{
Iron Pincer Complexes as Catalysts in Cross-coupling of Aryl Halides and Phenylboronic Acid
}

\author{
Lolakshi Mahesh Kumar, Badekai Ramachandra Bhat* \\ Catalysis and Materials Laboratory, Department of Chemistry, National Institute of Technology Karnataka, \\ Surathkal, Mangalore 575025, India. \\ *Corresponding author E-mail: ram@nitk.edu.in.
}

\begin{abstract}
Pincer complexes with iron as active metal center were synthesized to study their catalytic activity in Suzuki-Miyaura coupling reactions. Tridentate pincer ligand was synthesized by the reaction of diphenylchlorophosphine with $\mathrm{m}$-aminophenol and m-phenylenediamine respectively in a 2:1 ratio in the presence of triethylamine as a base and tetrahydrofuran as solvent media. The resultant ligand was complexed with $\mathrm{FeSO}_{4}$ to obtain PCP complexes, $\mathrm{C}-1$ with $\mathrm{O}$ and $\mathrm{N}$ atoms in the side arms and $\mathrm{C}-2$ with both $\mathrm{N}$ atoms in the side arms. The synthesized complexes were examined for their $\mathrm{C}-\mathrm{C}$ coupling efficiency in cross-coupling between phenyl boronic acid and para substituted halobenzenes. The research study aims to provide an alternative approach to the Pd catalyzed cross coupling methods, an otherwise subjugated method to obtain cross-coupled products. Also the study implores on the effect of variation in the side arm atom relating to the donating ability of the ligand and thereby relatively affecting the coupling yield of the catalysts.
\end{abstract}

Keywords: cross-coupling; PCP; Pincer complexes; Suzuki-Miyaura.

\section{Introduction}

Complexes bearing pincer ligands are a promising class of organometallic complexes that have been established and recognized as effective pre-catalysts for diverse reactions. Their unique metal bound structures, high thermal stability, and process viability makes them the most active catalysts for organic transformations involving the activation of bonds ${ }^{1}$. They are well known to catalyze Suzuki-Miyaura cross-coupling reaction with high catalytic activity and selectivity ${ }^{2-5}$.

However, the most emphasized complexes catalyzing this coupling reaction are with palladium, which despite having unsurpassed catalytic activity is less abundant and expensive ${ }^{6-9}$. The metals like nickel ${ }^{10-11}$ and ruthenium ${ }^{12-13}$ are also employed which show good activity, but deters the green motive of catalysis by being environmentally less benevolent.

Therefore, the study and development of catalytic reactions promoted by inexpensive and environmentally benign metals becomes a very important and needful catalytic approach. Our present work considers both the metal price and cost of ligand synthesis and has focused on the iron complexes with a PCP-pincer ligand. Iron is widely studied for its catalytic potential due to its low cost and low toxicity ${ }^{14-16}$. However, its reactivity as pincer complexes is a less explored field ${ }^{17-18}$ which we intend to study and apply in catalyzing Suzuki coupling reactions.

\section{Materials and Method}

Ferrous sulphate heptahydrate was purchased from Merck, India and used as received. Other chemicals like m-aminophenol, mphenylenediamine, chlorodiphenylphosphine, tetrahydrofuran
(THF), triethylamine $\left(\mathrm{Et}_{3} \mathrm{~N}\right)$, acetonitrile $(\mathrm{ACN})$, phenylboronic acid and aryl halides were purchased from Sigma-Aldrich and used without further purification.

The ligands were synthesized as per the reported procedure ${ }^{19}$ with certain modifications. In a typical synthesis process, triethylamine $(1.85 \mathrm{~g}, 18.3 \mathrm{mmol})$ was dissolved in THF $(20 \mathrm{~mL})$ with the respective amine $(1 \mathrm{~g}, 9.2 \mathrm{mmol})$. The mixture was cooled to $0^{\circ} \mathrm{C}$ followed by drop wise addition of chlorodiphenylphosphine (4.04 $\mathrm{g}, 18.3 \mathrm{mmol}$ ) under stirring. The solution was brought to room temperature and refluxed overnight. The solution was then filtered, washed with anhydrous hexane $(2 \times 10 \mathrm{~mL})$, and the solvent was removed under vacuum to afford the ligands.

Ferrous sulphate was refluxed for $4 \mathrm{~h}$ with the ligands in $1: 1$ ratio in THF. The precipitates obtained were filtered and washed with ether (Scheme 1).

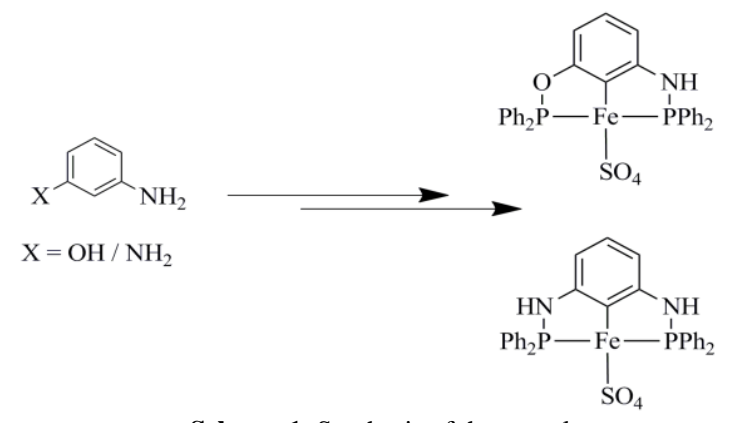

Scheme 1: Synthesis of the complexes

For characterization, complexes were studied under Bruker Alpha FTIR, Analytik Jena Specord UV Vis Spectrophotometer, Bruker AV 400 NMR spectroscopy, Sherwood UK magnetic susceptibility instrument. The catalysis reaction product analysis was carried 
out using Gas Chromatography (GC) (Shimadzu 2014, Japan); the instrument has a 5\% diphenyl and 95\% siloxane Restek capillary column (30 m length and $0.25 \mathrm{~mm}$ diameter) and Flame Ionization Detector (FID).

\section{Results and Discussion}

FTIR analysis of the ligands L-1 and L-2 showed medium intensity secondary $\mathrm{N}-\mathrm{H}$ stretching at 3350 and $3360 \mathrm{~cm}^{-1}$ respectively indicating the bond formation between $\mathrm{N}$ and P. Formation of bond between $\mathrm{O}$ and $\mathrm{P}$ in $\mathrm{L}-1$ is indicated by the absence of broad peak for O-H stretching and appearance of a peak at $750 \mathrm{~cm}^{-1}$ for $\mathrm{P}-\mathrm{O}$ stretching. A sharp peak at $694 \mathrm{~cm}^{-1}$ is assigned to the P-N stretching in both the ligands and the complexes ${ }^{20}$. (Fig. 1)
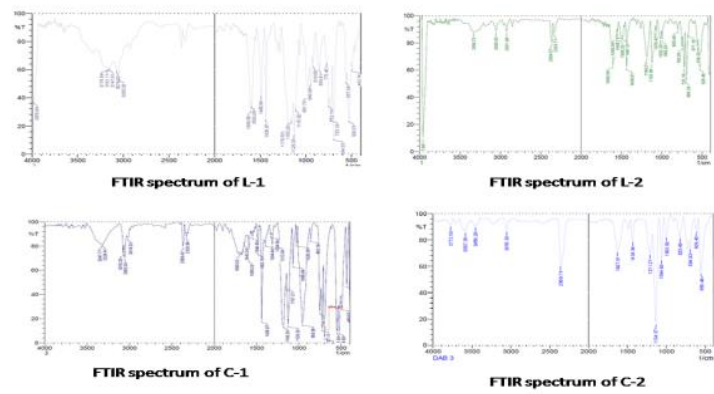

Figure 1: FTIR spectra of ligands and the complexes

Electronic spectra of the complexes along with their corresponding ligands were recorded as a confirmatory measure to imply the complex formation (Fig. 2). In all the spectra recorded, there is a shift in the absorption band from the ligand to the complex perceptibly indicating the complex formation. The absorption bands observed in the spectrum of the free ligand have shifted to lower energy region in the spectra of complexes due to the coordination of the ligand with metal ion. All the transitions are majorly due to intra ligand charge transfer transitions (ILCT) and ligand to metal charge transfer transitions (LMCT).
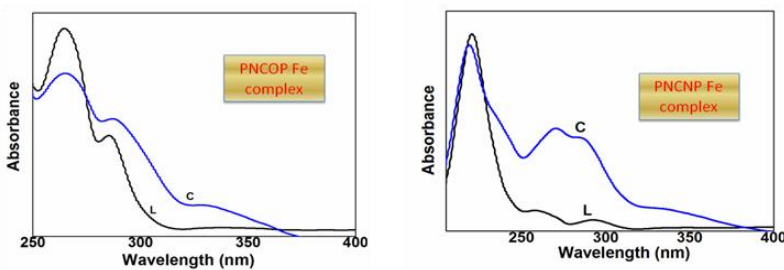

Figure 2: Electronic spectra of ligands and the complexes

Magnetic moments for C-1 and C-2 were observed to be $1.82 \mathrm{BM}$ and $1.93 \mathrm{BM}$ indicating paramagnetism corresponding to one unpaired electron and thereby supporting the formation of $\mathrm{Fe}(\mathrm{III})$ complexes $^{21}$.

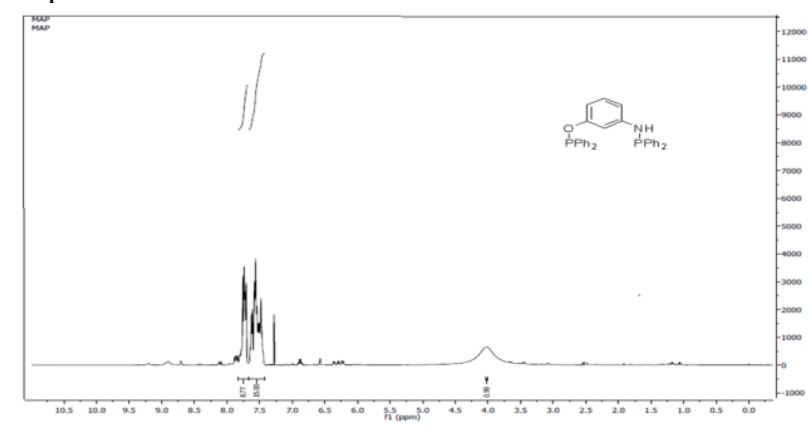

$87.89-7.75(m, 9 H), 7.65-7.43(m, 15 H), 4.02($ br s, $1 \mathrm{H}, \mathrm{NH})$

(a) ${ }^{1} \mathrm{H}$ NMR spectrum of ligand L-1

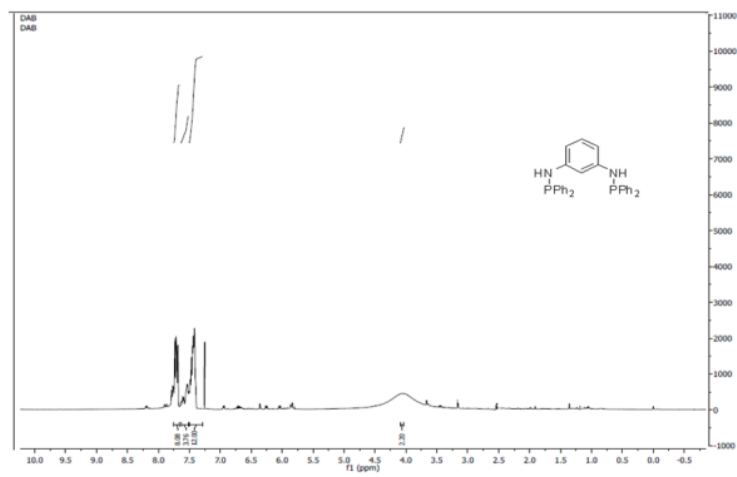

$87.82-7.62(\mathrm{~m}, 8 \mathrm{HH}), 7.61-7.54(\mathrm{~m}, 4 \mathrm{H}), 7.53-7.40(\mathrm{~m}, 12 \mathrm{H}), 4.02(\mathrm{brs}, 2 \mathrm{H}, \mathrm{NH})$

(b) ${ }^{1} \mathrm{H}$ NMR spectrum of ligand $\mathrm{L}-2$

Figure 3: ${ }^{1} \mathrm{H}$ NMR spectra of ligands (a) L-1 (b) L-2

The significant conversion of biaryls was determined by Gas Chromatography (GC). The reaction between 4-bromobenzonitrile and phenyl boronic acid was chosen as a model reaction to evaluate the catalytic activity of the catalyst precursors. Various factors including solvent, catalyst loading, base, temperature and time were screened to optimize the reaction conditions (Fig. 4)

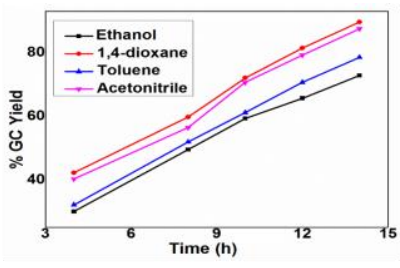

Effect of solvent on catalytic activity of C-2

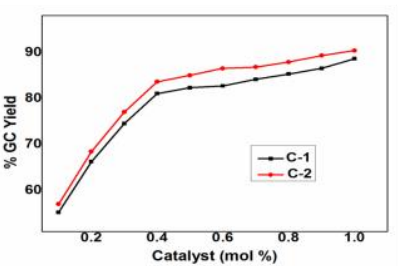

Effect of catalyst concentration

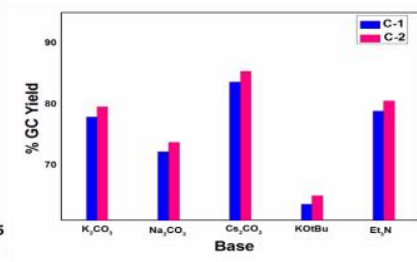

Effect of base on catalytic activity

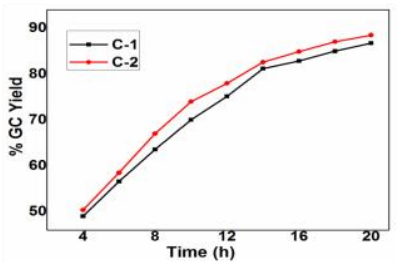

Effect of reaction time on catalytic activity
Figure 4: Optimization studies of the catalysts

The promising results were a motivation to extend the cross coupling reaction between phenyl boronic acid and different aryl halides using the optimized reaction conditions. The results of these reactions are summarized in Table 1.

Table 1: Catalytic activity study for complexes

\begin{tabular}{|c|c|c|c|c|}
\hline \multirow{2}{*}{ Entry } & \multirow{2}{*}{$\mathrm{X}$} & \multirow{2}{*}{$\mathrm{R}$} & \multicolumn{2}{|c|}{ Yield $(\%)^{\mathrm{a}}$} \\
\hline & & & $\mathrm{C}-1$ & $\mathrm{C}-2$ \\
\hline 1 & \multirow{7}{*}{$\mathrm{Br}$} & $\mathrm{H}$ & 59 & 61 \\
\hline 2 & & $\mathrm{CN}$ & 85 & 89 \\
\hline 3 & & $\mathrm{OCH}_{3}$ & 74 & 76 \\
\hline 4 & & $\mathrm{COCH}_{3}$ & 80 & 87 \\
\hline 5 & & $\mathrm{NHCOCH}_{3}$ & 78 & 83 \\
\hline 6 & & $\mathrm{~F}$ & 40 & 42 \\
\hline 7 & & $\mathrm{CH}_{3}$ & 57 & 60 \\
\hline 8 & \multirow{2}{*}{ I } & $\mathrm{OH}$ & 69 & 74 \\
\hline 9 & & $\mathrm{CN}$ & 89 & 94 \\
\hline 10 & $\mathrm{Cl}$ & $\mathrm{CHO}$ & 67 & 71 \\
\hline
\end{tabular}

Reaction conditions: Aryl halide (1.0 mmol), Phenyl boronic acid (1.3 $\mathrm{mmol}), \mathrm{Cs}_{2} \mathrm{CO}_{3}(2.0 \mathrm{mmol})$, catalyst $(0.4 \mathrm{~mol} \%)$, solvent $(5.0 \mathrm{~mL}), 14 \mathrm{~h}$.

${ }^{a} \mathrm{GC}$ yields, average of 3 trials. 


\section{Conclusions}

In view of the current use of expensive Pd catalyst for crosscoupling reactions, an attempt was made to synthesize more economical transition metal, iron based pincer complexes as an alternative catalyst. Various molecular characterizations showed the successful synthesis of the desired pincer complexes. The iron catalysts were found to catalyze the coupling reactions very effectively. The effect of reaction parameters like solvent, alkalinity, reaction time, and catalyst loading suggested that among the tested factors, the cross-coupling reaction showed better performance in ACN solvent with $\mathrm{Cs}_{2} \mathrm{CO}_{3}$ base and a reaction time of $14 \mathrm{~h}$ with for $0.4 \mathrm{~mol} \%$ of the synthesized catalysts. The electron withdrawing groups on the aryl halides accelerated the process of conversion to biaryls whereas presence of electron donating groups brought about lesser conversion. Among the halides, iodide was observed to be a better leaving group. Complex, C-2 with additional ' $\mathrm{N}$ ' donor atom in the side arm catalyses the coupling reaction better than $\mathrm{C}-1$.

\section{Acknowledgement}

The author, Mrs. Lolakshi Mahesh Kumar would like to acknowledge National Institute of Technology for extending the research fellowship.

\section{References}

[1] Morales-Morales D and Jensen CGM., The Chemistry of Pincer Compounds, Elsevier, 2011.

[2] Rosa GR, Rosa CH, Rominger F, Dupont J \& Monteiro AL (2006), A mixed NCP pincer palladacycle as catalyst precursor for the coupling of aryl halides with aryl boronic acids. Inorganica chimica acta, 359, 6, 1947-1954.

[3] Zhang BS, Wang C, Gong JF \& Song MP (2009), Facile synthesis of achiral and chiral PCN pincer palladium(II) complexes and their application in the Suzuki and copper-free Sonogashira crosscoupling reactions. Journal of Organometallic Chemistry, 694, 16, 2555-2561.

[4] Yorke J, Sanford J, Decken A \& Xia A (2010), Iminophosphinite pincer palladium complexes: Synthesis and application. Inorganica Chimica Acta, 363, 5, 961-966.

[5] Li P, Zhou HF, Liu F, Hu ZX \& Wang HX (2013), NCN palladium pincer via transmercuration. Synthesis of [2-(2-oxazoliny)-6-(2pyridyl)] phenylpalladium(II) chloride and its catalytic activity in Suzuki coupling. Inorganic Chemistry Communications, 32, 78-81.

[6] Kumaravel M, Kumar P \& Balakrishna MS (2014), Application of bisphosphomide-palladium(II) pincer complex in Suzuki-Miyaura cross-coupling reaction under microwave irradiation. Journal of Chemical Sciences, 126, 3, 711-716.

[7] Selander N \& Szabó KJ (2011), Catalysis by Palladium Pincer Complexes. Chemical Reviews, 111, 3, 2048-2076.

[8] Singleton JT (2003), The uses of pincer complexes in organic synthesis. Tetrahedron, 59,11, 1837-1857.

[9] Hurtado J, Ibañez A, Rojas R \& Valderrama M (2010), Palladium(II) complexes bearing the new pincer ligand 3,5-bis(indazol-2ylmethyl)toluene; Synthesis and catalytic properties. Inorganic Chemistry Communications, 13, 9, 1025-1028.

[10] Inamoto K, Kuroda J, Hiroya K, Noda Y, Watanabe M \& Sakamoto T (2006), Synthesis and Catalytic Activity of a Pincer-Type Bis(imidazolin-2-ylidene) Nickel(II) Complex. Organometallics, 25, 12, 3095-3098.

[11] Estudiante-Negrete F, Hernández-Ortega S \& Morales-Morales D (2012), Ni(II)-POCOP pincer compound [NiCl\{C10H5-2,10(OPPh2)2\}] an efficient and robust nickel catalyst for the SuzukiMiyaura coupling reactions. Inorganica Chimica Acta, 387, 58-63.

[12] Kumar A, Agarwal M, Singh AK \& Butcher RJ (2009), Palladium(II), platinum(II), ruthenium(II) and mercury(II) complexes of potentially tridentate Schiff base ligands of $(\mathrm{E}, \mathrm{N}, \mathrm{O})$ type $(\mathrm{E}=\mathrm{S}$, $\mathrm{Se}, \mathrm{Te})$ : Synthesis, crystal structures and applications in Heck and Suzuki coupling reactions. Inorganica Chimica Acta, 362, 9, 32083218.
[13] Na Y, Park S, Han SB, Han H, Ko S \& Chang S (2004), Ruthenium-Catalyzed Heck-Type Olefination and Suzuki Coupling Reactions: Studies on the Nature of Catalytic Species. Journal of the American Chemical Society, 126, 1, 250-258.

[14] Plietker B, Iron Catalysis in Organic Chemistry: Reactions and Applications, John Wiley \& Sons, 2008.

[15] Bolm C, Legros J, Le Paih J \& Zani L (2004), Iron-catalyzed reactions in organic synthesis. Chemical reviews 104, 12, 6217-6254.

[16] Enthaler S, Junge K \& Beller M (2008), Sustainable Metal Catalysis with Iron: From Rust to a Rising Star? Angewandte Chemie International Edition, 47, 18, 3317-3321.

[17] Bichler B, Holzhacker C, Stöger B, Puchberger M, Veiros L. F \& Kirchner K (2013), Heterolytic Cleavage of Dihydrogen by an Iron (II) PNP Pincer Complex via Metal-Ligand Cooperation. Organometallics, 32, 15, 4114-4121.

[18] Bhattacharya P, Krause JA \& Guan H (2011), Iron Hydride Complexes Bearing Phosphinite-Based Pincer Ligands: Synthesis, Reactivity and Catalytic Application in Hydrosilylation Reactions. Organometallics, 30, 17, 4720-4729.

[19] Feng J \& Cai C (2013), An efficient synthesis of perfluoroalkenylated aryl compounds via Pincer-Pd catalyzed Heck couplings. Journal of Fluorine Chemistry, 146, 6-10.

[20] Shagidullin RR, Shakirov IK, Nuretdinova ON, Katsyuba SA \& Filippova EA (1985), Structural information content of the stretching vibration frequencies of $\mathrm{PN}$ and $\mathrm{PO}$ bonds in five-membered heterocyclic compounds. Bulletin of the Academy of Sciences of the USSR, Division of chemical science, 34, 10, 2064-2067.

[21] Barefield EK, Busch DH \& Nelson SM (1968), Iron, cobalt, and nickel complexes having anomalous magnetic moments. Quarterly Reviews, Chemical Society, 22, 4, 457-498. 